\title{
New Insights in to Molecular Therapeutic Mechanism of Statin Action in Heart Failure: Use of High-Throughput Transcriptome Analysis
}

\author{
Wada $\mathbf{A}^{*}$, Matsumoto T, Takayama T, Suzaki M, Taniguchi A, Hara M, Fujii M and Isono T
}

Kusatsu General Hospital Cardiovascular Disease and Heart Failure Center, 1660 Yabase, Kusatsu, Shiga, Japan

\begin{abstract}
Background and objective: The etiology of heart failure (HF) is multifactorial and a variety of mediators are involved in its progression. Thus, elucidation of transcriptome complexity and understanding the functions of differentially expressed genes, together with therapeutic molecular mechanisms underlying development of HF, are a major focus of HF research. A recent metaanalysis demonstrated that statin therapy reduced the risk of hospitalization and death due to HF.

Methods and results: We first performed global cardiac transcriptome analysis in a canine model of tachycardia-induced $\mathrm{HF}$ using next generation genome sequencers; secondly we evaluated the effects of a statin, pitavastatin $(0.3 \mathrm{mg} / \mathrm{kg}, \mathrm{n}=6), \mathrm{on}$ cardiac function and gene expressions in HF. A total of 426 genes were differentially expressed, 401 of which were up-regulated in failing heart compared to normals. We identified the most changed pathways which included angiopoietin receptor Tie2, $T$ cell receptor and CXCR4 signaling pathways. Despite an absence of differences in fractional shortening, pitavastatin significantly lowered elevations in left ventricular end-diastolic pressure and shortened prolongation of tau. Furthermore, pitavastatin suppressed up-regulation of expression of genes such as SRC, SHC1, VAV1, ELK1 and FLNA mRNA. Not only collagen types I and III, but also VI mRNA involved in maintaining structural integrity, were decreased compared with vehicle. These results indicate that statin may improve diastolic properties affecting cell proliferation, inflammation, oxidative stress, energy metabolism and extracellular matrix remodeling.
\end{abstract}

Conclusion: High-throughput transcriptome analysis can provide new insights into cellular mechanisms underlying HF and therapeutic use of statin for treatment of $\mathrm{HF}$.

Keywords: Transcriptome analysis; Heart failure; Statin; Next generation genome sequencers

\section{Introduction}

The etiology of heart failure (HF) is multifactorial and a variety of mediators are involved in its progression; therefore, the total spectrum of events responsible for development of HF is as yet unknown. Since the causes of cardiac dysfunction are likely to reflect alterations in cardiac protein expression, we have previously used a proteomic approach to investigate alterations in protein expression in a canine model of tachycardia-induced HF [1]. We compared two-dimensional electrophoresis protein patterns of left ventricular (LV) samples obtained from normal and failing myocardium and identified the altered undetermined protein spots. While proteomic analysis provided some important information on global changes in HF, only $\sim 1000$ differences in protein expression patterns between HF and normals were observed. Transcriptome sequencing is particularly useful in assessing the current state of a cell or tissue and the possible effects of disease states or treatment conditions on the transcriptome [2]. Deep mRNA sequencing analysis of cardiac mRNAs has demonstrated that a preponderance of mitochondrial transport, cytoskeletal and contractile genes are differentially expressed in failing heart [3]. We identified changes in gene expression strongly related to occurrence of HF using global transcriptome analysis of next-generation sequencers (NGS) and revealed that expression of inflammatory interleukin-related genes was enhanced in failing heart [4]. Cardiac myocytes can secret proinflammatory cytokines in response to stress and injury and HF is considered to be a chronic state of immune activation during which myocardial remodeling occurs via both altered myocardial contractility and extracellular matrix (ECM) accumulation [5]. Apart from reducing lipid concentration via HMG CoA reductase inhibition, statins exhibit pleiotropic activity exerting anti-inflammatory and antioxidant effects, inhibiting neurohormonal activation and benefiting myocardial reconstruction [6]. However, in HF patients with predominantly reduced ejection fraction (EF), two major prospective randomized controlled trials failed to show a mortality benefit with statin therapy
$[7,8]$. While such results were disappointing, benefits related to nonfatal HF hospitalization and mortality have recently been reported. In a meta-analysis evaluating the effect of statin therapy on HF events with over 132, 000 participants, such therapy modestly reduced the risk of non-fatal HF hospitalization and the composite outcome of HF death and non-fatal hospitalization [9]. Although statins possess certain cholesterol-independent effects that make them attractive candidates for HF pharmacotherapy, the overall mechanism by which statins lead to a reduced incidence of HF is not fully clear. The elucidation of transcriptome complexity in failing heart and understanding the underlying functions of various differentially expressed genes have become a major focus for post-genome research; thus high-throughput analysis can be used to evaluate the molecular mechanism of statin actions in HF. Transcriptome changes assessed by RNA sequencing (seq) play a valuable role in cardiovascular medicine, because such changes can identify how cardiovascular disease states undergo change with treatment.

In this study, we first applied the RNA-seq method to identify transcriptome-wide changes in cardiac gene expression in a canine model with HF, compared to control animals. Secondly we assessed the impact of therapeutic intervention using statin treatment on the functional significance of the categories of transcriptome whose expression was most highly up-regulated in HF.

*Corresponding author: Atsuyuki Wada, Kusatsu General Hospital Cardiovascular Disease and Heart Failure Center, 1660 Yabase, Kusatsu, Shiga, 525-8585, Japan Tel: 81-77-563-8866; Fax: 81-77-527-3037; E-mail: wada@belle.shiga-med.ac.jp

Received December 28, 2017; Accepted January 12, 2018; Published January 19, 2018

Citation: Wada A, Matsumoto T, Takayama T, Suzaki M, Taniguchi A, et al. (2018) New Insights in to Molecular Therapeutic Mechanism of Statin Action in Heart Failure: Use of High-Throughput Transcriptome Analysis. Cardiovasc Pharm Open Access 6: 232. doi: 10.4172/2329-6607.1000232

Copyright: ( 2018 Wada A, et al. This is an open-access article distributed under the terms of the Creative Commons Attribution License, which permits unrestricted use, distribution, and reproduction in any medium, provided the original author and source are credited. 
Citation: Wada A, Matsumoto T, Takayama T, Suzaki M, Taniguchi A, et al. (2018) New Insights in to Molecular Therapeutic Mechanism of Statin Action in Heart Failure: Use of High-Throughput Transcriptome Analysis. Cardiovasc Pharm Open Access 6: 232. doi: 10.4172/23296607.1000232

Page 2 of 6

\section{Materials and Methods}

\section{Animal and sample preparation}

All animal experiments were conducted according to the Guide for the Care and Use of Laboratory Animals (Department of Health and Human Services, National Institutes of Health, Publication no. 86-23) and approved by the Committee of the Research Center for Animal Life Science at Shiga University of Medical Science (Approval No. 2003-716). After anaesthesia was induced using pentobarbital sodium $(25 \mathrm{mg} /$ $\mathrm{kg}$ ), animal preparation was performed and HF was induced by rapid right ventricular pacing ( 240 beats per minute, 28 days) in beagles (Kitayama Labes Co, Ltd, Japan), as described previously [10,11].

\section{Experimental protocol and effects of statin on hemodynamics in $\mathrm{HF}$}

Animals were divided into three groups in a randomized fashion:

- The statin group $(\mathrm{n}=6)$ received pitavastatin $(0.3 \mathrm{mg} / \mathrm{kg} /$ day, orally once daily).

- The HF group $(n=6)$ received only vehicle.

- The normal group $(n=6)$ underwent the same procedure without pacing.

We selected a dosage of the potent long-acting HMG-CoA reductase inhibitor pitavastatin that appeared to ameliorate vascular endothelial dysfunction in HF, as reported previously [12]. Drug treatment commenced on the first day of pacing and continued for four weeks. On the 29th day after initiation of pacing, pacemakers were deactivated and hemodynamic and echocardiographic measurements were performed to estimate left ventricular (LV) chamber diameter and fractional shortening (FS) during a brief cessation of rapid pacing; serum cholesterol levels were also measured as previously reported [12]. Subsequently, all animals were treated with thiopental sodium to provide conscious sedation, a micro-manometer catheter was placed into the LV and LV end-diastolic pressure (LVedp) and the time constant of LV pressure decay (tau) were measured as previously reported [13]. Animals were deeply anaesthetized and euthanized by bleeding. The heart was rapidly removed and transmural sections of the LV anterior free wall were frozen in liquid nitrogen.

\section{RNA preparation and genome analyzer sequencing}

Total RNA was extracted from frozen LV muscle of the three groups using acid guanidinium thiocyanate-phenol-chloroform. Total RNA was quantified with Agilent 2100 bio analyzer (Agilent) following the manufacturer's instructions. Template molecules for high throughput DNA sequencing were prepared from total RNA using the mRNA-Seq Sample Preparation Kit (Illumina) as described previously [4]. The library was quantified with Agilent 2100 bio analyzer and sequencing was performed on a Genome Analyzer GAIIx with 37 cycles, using Cycle Sequencing v4 regents (Illumina) [4].

Sequence data sets: Human genome builds 19 (hg19) was downloaded from University of California Santa Cruz genome browser (http://genome.ucsc.edu/). We used hg19 in place of canine genome build 2 (canFam2) as indicated in our previous studies [4].

Data analysis: Reads were aligned using ELAND v2 of CASAVA Software 1.7 with the sequence data sets. The data were viewed using Genome Studio Software (Illumina). Advanced analysis for detecting significant pathways was performed using Avadis NGS software (Strand Scientific Intelligence Inc., San Francisco, CA). Genes with significantly differing expression levels were identified by the fold change method (Fold change $>2$ ), statistically analyzed by BenjaminHochberg's FDR $(\mathrm{P}<0.05)$ and categorized into particular pathway categories by Find Significant Pathway analysis $(\mathrm{P}<0.05)$, Quantitative reverse-transcription-polymerase chain reaction (qRT-PCR).

Quantitative RT-PCR was performed using the Light Cycler 480 SYBG Master I Mix and Light Cycler 480 System II (Roche Diagnostics, Germany) as described previously [4]. Gene expression was normalized using the GAPDH gene. Canine-specific primer sequences are listed in Table 1. All quantification analyses were performed in triplicate.

Total cholesterol analysis: Serum total cholesterol levels were determined using commercially available kits (Wako Pure Chemicals, Japan) as described previously [12].

Histomorphometric analysis: Picrosirius red staining was performed to evaluate the degree of fibrosis in each sampled LV as previously reported [14].

Statistical analysis: Data are expressed as mean \pm SEM. Differences between two groups were assessed by Student's t test when appropriate. Differences among three groups were assessed by ANOVA with Scheffe's-F test when appropriate. A P value of 0.05 was chosen as the cut-off significance level.

\section{Results}

\section{Cardio-hemodynamics and blood analysis}

Cardio-hemodynamic changes and statin effects are summarized in Table 2. After four weeks of rapid pacing, mean arterial pressure, cardiac output, $\%$ fractional shortening and $\mathrm{dP} / \mathrm{dt}$ max decreased and LV end-diastolic dimensions increased compared with the normal group. These data suggest that chronic rapid pacing produced severe HF. Although there were no significant differences in these values between the HF and statin groups, pitavastatin significantly lowered the elevated LVedp and shortened the prolongation of tau. Total cholesterol levels in HF were significantly lower than the normal group,

\begin{tabular}{c|c|c|}
\hline Gene & Forward (5' to $\mathbf{3}^{\prime}$ ) & Reverse (5' to $\mathbf{3}^{\prime}$ ) \\
\hline ELK1 & GCCTCTATTCCACCTTCACCATC & CTTGGACTGGTGCTCCTGCTC \\
FLNA & CCAAGCTGAAGCCAGGGGCTC & TCCACGTACACCAGCACCTCTC \\
SHC1 & TTCCTACTTGGTTCGGTACATGG & GGGCGGCTACAGGGCTTTCTC \\
SRC & TCCAGATTGTCAACAACACAGAGG & GACTCCCGTCTGGTGATCTTGC \\
VAV1 & ACCCTGTCTGCTCTGTCCTGG & CACGCAGTCATACAGGTCCTC \\
COL1A1 & GGCACCCCTGGAAGGGACG & TGGAGGCCCTTGGGGTCCTG \\
COL3A1 & CCAAAAGGTGACAAGGGTGAC & CATCCCCTGAAATCCAGGTTCAC \\
COL6A1 & CTGAGCATCATCGCCACGGAC & AGGAGCAGCACACTTGCTCCAC \\
GAPDH & GTCATCATCTCTGCTCCTTCT & CTCAGGGATGACCTTGCCCAC \\
\hline
\end{tabular}

Table 1: Oligonucleotides used for qRT-PCR.

\begin{tabular}{|c|c|c|c|}
\hline & Normal $(n=6)$ & Vehicle $(n=6)$ & Statin $(n=6)$ \\
\hline $\begin{array}{c}\text { Mean arterial pressure } \\
(\mathrm{mmHg})\end{array}$ & $122.0 \pm 7.6$ & $109.8 \pm 4.0^{*}$ & $112.6 \pm 3.7^{*}$ \\
\hline Cardiac output (L/min) & $3.06 \pm 0.21$ & $1.38 \pm 0.16^{\star}$ & $1.44 \pm 0.24$ \\
\hline LVedp (mmHg) & $6.6 \pm 1.4$ & $29.5 \pm 3.3^{*}$ & $23.5 \pm 2.7^{* \dagger}$ \\
\hline $\mathrm{dP} / \mathrm{dt} \max (\mathrm{mmHg} / \mathrm{sec})$ & $5132.6 \pm 290.7$ & $1829.1 \pm 287.5^{*}$ & $\begin{array}{c}1995.1 \pm \\
111.1^{\star}\end{array}$ \\
\hline tau (msec) & $23.4 \pm 2.4$ & $40.1 \pm 2.6^{*}$ & $31.6 \pm 2.5^{* \dagger}$ \\
\hline LVDd (mm) & $30.8 \pm 1.6$ & $39.7 \pm 2.2^{*}$ & $38.9 \pm 2.6^{*}$ \\
\hline$\%$ Fractional shortening & $28.3 \pm 3.1$ & $9.9 \pm 1.6^{*}$ & $13.8 \pm 2.7^{*}$ \\
\hline Total cholesterol, mg/dl & $141 \pm 10$ & $116 \pm 9^{*}$ & $111 \pm 8^{\star}$ \\
\hline
\end{tabular}

LVedp indicates LV end-diastolic pressure; LVDd; LV diastolic dimension. Values are mean \pm SEM. ${ }^{*} P<0.05$ compared with the normal group, $\dagger \mathrm{P}<0.05$ compared with the vehicle group

Table 2: Effects of pitavastatin on hemodynamics, LV geometry, LV function and blood analysis. 
Citation: Wada A, Matsumoto T, Takayama T, Suzaki M, Taniguchi A, et al. (2018) New Insights in to Molecular Therapeutic Mechanism of Statin Action in Heart Failure: Use of High-Throughput Transcriptome Analysis. Cardiovasc Pharm Open Access 6: 232. doi: 10.4172/23296607.1000232

Page 3 of 6

however, the values were not different between vehicle and statintreated groups.

\section{RNAseq Analysis of failing hearts}

We performed digital transcriptome analysis to study gene expression in LV muscle of normal and HF animals. Approximately 22 million reads and 15000 genes which aligned to the hg19 database were obtained. We performed digital transcriptome analysis to study gene expression in LV muscle of normal and HF animals. Comprehensive new gene data has been deposited in the DDBJ under accession number DRA005850, PRJDB5821 and SAMD00081461-69. A total of 426 genes were differentially expressed in failing heart compared to normal controls. Of these, 401 genes were significantly up-regulated whereas 25 were down-regulated. We thus turned our attention to the subsets of up-regulated genes expression in HF and Find Significant Pathway analysis was performed. As shown in Table 3, we identified the significantly changed pathways and selected the most principal three of these which were categorized into (1) Angiopoietin receptor Tie2 (Ang-Tie2), (2) T cell receptor (TCR) signaling in CD4, (3) chemokine (C-X-C motif) receptor 4 (CXCR4) pathways and listed the genes among the three pathways (Table 4 ).

\begin{tabular}{|c|c|c|c|}
\hline Pathway & $\begin{array}{l}\text { Matched with } \\
\text { technology }\end{array}$ & $\begin{array}{c}\text { Matched } \\
\text { with entity } \\
\text { list }\end{array}$ & P-Value \\
\hline $\begin{array}{l}\text { Angiopoietin receptor Tie2-mediated } \\
\text { signaling }\end{array}$ & 38 & 6 & $6.93 \mathrm{E}-06$ \\
\hline TCR signaling in naive $\mathrm{CD}^{4+} \mathrm{T}$ cells & 103 & 9 & $9.82 \mathrm{E}-06$ \\
\hline CXCR4-mediated signaling events & 130 & 9 & $5.72 \mathrm{E}-05$ \\
\hline PDGFR-beta signaling pathway & 37 & 5 & 1.03E-04 \\
\hline $\begin{array}{l}\text { Transcriptional activation of cell cycle } \\
\text { inhibitor p21 }\end{array}$ & 2 & 2 & 1.45E-04 \\
\hline $\begin{array}{l}\text { Signaling events mediated by HDAC } \\
\text { Class III }\end{array}$ & 23 & 4 & $1.54 \mathrm{E}-04$ \\
\hline EPHB forward signaling & 24 & 4 & 1.83E-04 \\
\hline S1P1 pathway & 43 & 5 & 2.05E-04 \\
\hline $\begin{array}{c}\text { Signaling events mediated by HDAC } \\
\text { Class I }\end{array}$ & 78 & 6 & 4.22E-04 \\
\hline Cell-extracellular matrix interactions & 13 & 3 & 4.56E-04 \\
\hline $\begin{array}{c}\text { Signaling events mediated by VEGFR1 } \\
\text { and VEGFR2 }\end{array}$ & 50 & 5 & 4.45E-04 \\
\hline E-cadherin signaling events & 14 & 3 & 7.13E-04 \\
\hline TCR signaling in naive $\mathrm{CD}^{8+} \mathrm{T}$ cells & 83 & 6 & 7.90E-04 \\
\hline Regulation of nuclear SMAD2/3 signaling & 191 & 9 & 8.01E-04 \\
\hline Cell junction organization & 39 & 4 & 0.00149 \\
\hline Signaling events mediated by TCPTP & 68 & 5 & 0.00159 \\
\hline Thromboxane A2 receptor signaling & 20 & 3 & 0.00171 \\
\hline C-MYB transcription factor network & 73 & 5 & 0.00203 \\
\hline Alpha4 beta1 integrin signaling events & 21 & 3 & 0.00227 \\
\hline Role of DCC in regulating apoptosis & 7 & 2 & 0.00292 \\
\hline Protein folding & 7 & 2 & 0.00293 \\
\hline Cell junction organization & 25 & 3 & 0.00329 \\
\hline IL2-mediated signaling events & 80 & 5 & 0.00353 \\
\hline $\begin{array}{l}\text { Fc-epsilon receptor I signaling in mast } \\
\text { cells }\end{array}$ & 51 & 4 & 0.00384 \\
\hline $\begin{array}{l}\text { Caspase-mediated cleavage of } \\
\text { cytoskeletal proteins }\end{array}$ & 8 & 2 & 0.00387 \\
\hline $\begin{array}{l}\text { Regulation of cytoskeletal remodeling } \\
\text { and cell spreading }\end{array}$ & 8 & 2 & 0.00388 \\
\hline IL4-mediated signaling events & 54 & 4 & 0.00439 \\
\hline Transport of organic anions & 9 & 2 & 0.00495 \\
\hline
\end{tabular}

Table 3: The significantly up-regulated representative gene pathways in HF identified by Find Significant pathway analysis.

\section{Verification of gene expression by qRT-PCR analysis}

Next, to validate the RNAseq data from Find Significant Pathway analysis and to investigate how statin therapy affected transcriptome expression among these three gene pathways, we selected the following five genes: ETS transcription factor 1 (ELK1), Shc1 adaptor protein (SHC1), filamin A (FLNA), vav guanine nucleotide exchange factor 1 (VAV1) and steroid receptor coactivators (SRC); this was done because expression of these genes overlapped within at least two transcriptome pathways. We performed qRT-PCR to confirm changes in mRNA expression. In these confirmative analyses, all of the selected genes were up-regulated in HF compared to normals; furthermore, pitavastatin suppressed activation of these genes compared to the HF group as shown in Figure 1. Therefore, our results showed that statin may exert therapeutic action in HF by causing changes in the levels of these highly expressed genes.

\begin{tabular}{|c|c|c|c|c|}
\hline $\begin{array}{c}\text { Angiopoietin } \\
\text { receptor Tie2- } \\
\text { mediated } \\
\text { signaling }\end{array}$ & $\begin{array}{c}\text { TCR signaling } \\
\text { in naive CD } \\
\text { cells }\end{array}$ & $\begin{array}{c}\text { CXCR4- } \\
\text { mediated } \\
\text { signaling } \\
\text { events }\end{array}$ & P-Value & $\begin{array}{c}\text { Fold } \\
\text { change }\end{array}$ \\
\hline \multirow{2}{*}{ CDKN1A } & - & - & 0.003752 & 4.24 \\
\cline { 2 - 5 } & DBNL & DBNL & 0.00072 & 2.47 \\
\hline \multirow{2}{*}{ ELK1 } & ELK1 & ELK1 & 0.00310 & 2.97 \\
\hline \multirow{2}{*}{ NOS3 } & FSCN1 & FLNA & 0.00158 & 3.05 \\
\hline PLD2 & - & - & 0.01242 & 4.14 \\
\hline \multirow{2}{*}{ PXN } & - & PXN & 0.01108 & 2.119 \\
\hline \multirow{2}{*}{ SHC1 } & SH3BP2 & SH3BP2 & 0.01727 & 3.40 \\
\hline & SHC1 & SHC1 & 0.00570 & 2.09 \\
\hline & SRC & SRC & 0.00097 & 2.74 \\
\hline & VAV1 & VAV1 & 0.04134 & 4.38 \\
\hline & WAS & WAS & 0.04637 & 2.99 \\
\hline
\end{tabular}

Table 4: Lists of genes showing a two-fold increase of expression in three highscoring pathways in HF.
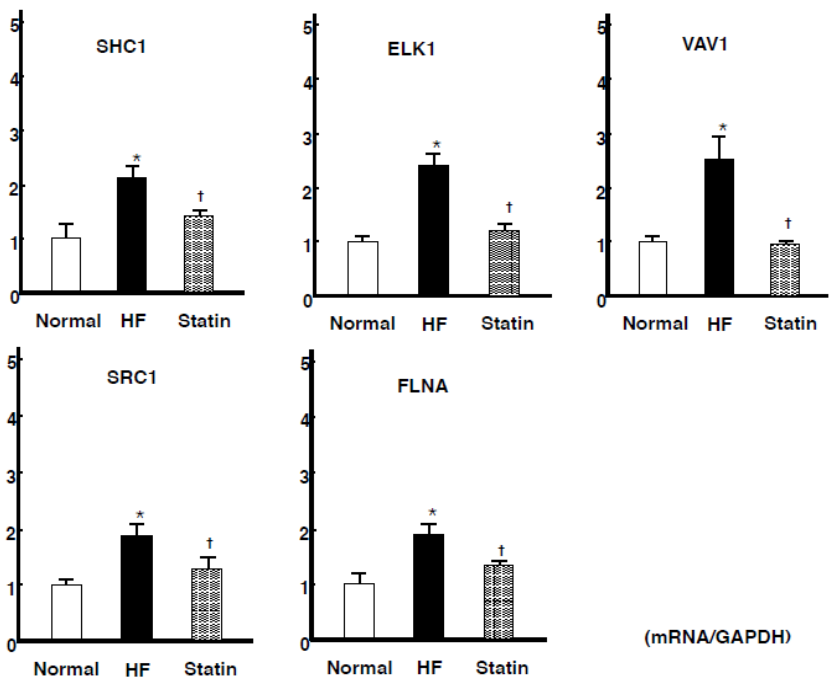

(mRNA/GAPDH)

Figure 1: qRT-PCR analysis of selected genes involved in the Ang-Tei2, TCR and CXCR4 signaling pathways in HF. Gene expression was normalized using the GAPDH gene in normal samples. Error bars represent standard errors from three experiments. ${ }^{*} \mathrm{P}<0.05$ compared with the normal group, ${ }^{\dagger} \mathrm{P}<0.05$ compared with the vehicle group. 
Citation: Wada A, Matsumoto T, Takayama T, Suzaki M, Taniguchi A, et al. (2018) New Insights in to Molecular Therapeutic Mechanism of Statin Action in Heart Failure: Use of High-Throughput Transcriptome Analysis. Cardiovasc Pharm Open Access 6: 232. doi: 10.4172/23296607.1000232

Page 4 of 6

\section{Effects of statin on cardiac fibrosis in HF}

Collagen volume fraction and collagen deposition are increased and myocardial fibrosis contributes to diastolic dysfunction in $\mathrm{HF}$ [14]. Chronic rapid pacing caused accumulation of a patchy collagen network and increased the extent of interstitial fibrosis in this study. The total amount of collagen deposits in the LV evaluated with picrosirius red stain are shown in Figure 2. In addition, pitavastatin significantly decreased fibrosis, expressed as the \% collagen volume fraction compared to the vehicle. We next investigated fibrosis-related gene expression in failing heart. NGS analysis revealed increases not only in collagen type I alpha 1 chain (COL1A1) and collagen type III alpha 1 chain (COL3A1), but also in collagen type VI alpha 1 chain (COL6A1) expression in HF and pitavastatin significantly reduced the levels of these mRNAs as shown in Figure 3.

\section{Discussion}

Identification of HF-associated gene pathways is essential to elucidate the etiology of this disease and to provide insight into the evaluation of molecular mechanisms underlying medical treatments for HF. In the present study, NGS analysis identified 426 genes whole expression was markedly changed in the tachycardia-induced failing heart. Furthermore, Find Significant Pathway analysis clarified the

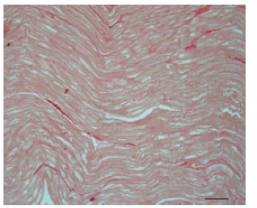

Normal(bar=50 $\mu \mathrm{m})$

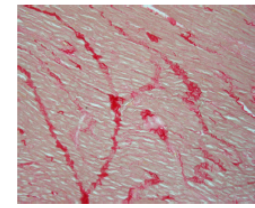

HF

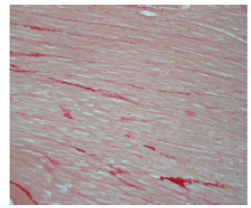

Statin

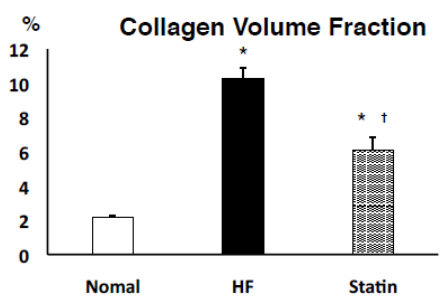

Figure 2: Upper panel-Representative examples of Picrosirius red staining in the LV of normal, vehicle and HF animals treated with statin. Original magnification $\times 200$. Scale bar indicates $50 \mu \mathrm{m}$.

Lower panel-Quantification of collagen volume fraction in LV of normal (empty bars), vehicle (HF, solid bars), and pitavastatin-treated HF (Statin, shaded bars). ${ }^{*} \mathrm{P}<0.05$ vs. normal animals, ${ }^{\dagger} \mathrm{P}<0.05$ vs. HF group.
COL1A

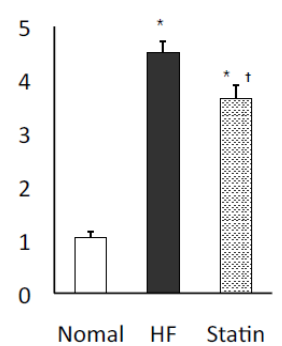

COL3A1

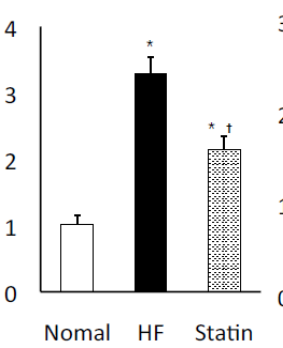

COL6A1

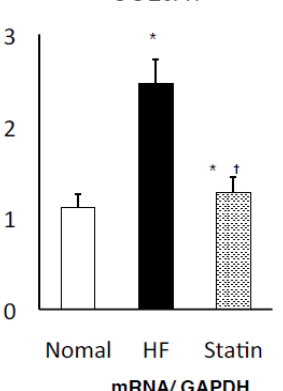

Figure 3: qRT-PCR analysis of collagen type I and III, VI mRNAs in LV treated with statin in HF. Gene expression was normalized using the GAPDH gene in normal samples. ${ }^{*} \mathrm{P}<0.05$ vs. normal animals, ${ }^{+} \mathrm{P}<0.05$ vs. HF group. most up-regulated gene pathways differentially expressed between HF and normal controls. These included the Ang-Tie2, TCR and CRCX4 signaling pathways, suggesting that they may play critical roles in the progression of HF. After searching for the most important gene pathways, we investigated whether stain therapy would affect expression of these HF-related transcriptomes. Although it did not change systolic dysfunction, pitavastatin improved diastolic properties, as exhibited by a lower LVedp and shortening of tau compared to vehicle. We next performed qRT-PCR to evaluate changes in the five core genes, $\mathrm{SHC1}$, VAV1, SRC, FLNA and ELK-1, which may participate in molecular and structural LV remodeling; pitavastatin significantly suppressed activation of expression of these genes. We also found that pitavastatin significantly decreased collagen type I, III and VI gene expression and cardiac collagen deposition compared with vehicle. The effects of statin on LV diastolic properties in HF can be distinguished, not only at the level of cardiac hemodynamics, but also at cellular and molecular levels.

Two large scale trials did not show that statins improved survival in HF with reduced ejection fraction $(\mathrm{EF})[7,8]$, however, randomized controlled trials with statins demonstrated reduced numbers of first HF hospitalizations and the composite of first non-fatal HF hospitalization or HF death [9]. In particular, in patients with HF preserved EF (HFpEF), statin use was associated with reduced all-cause mortality and combined all-cause mortality or cardiovascular hospitalization in the Swedish Heart Failure Registry [15]. Identification of genes differentially expressed between HF and normal controls is of help in understanding the molecular mechanisms underlying this disease; furthermore it is important in elucidating the extent to which statin affected transcriptome expression in failing hearts using NGS analysis.

Angiopoietins are endothelial growth factors binding to the Tie2 receptor and the Ang-Tie2 system is important for cardiac, blood vascular development and regulates angiogenesis and pathological endothelial inflammation [16]. A number of angiopoietin family members have been identified, the most important of which are angiopoietin 1 and angiopoietin 2, plasma angiopoietin 2 levels have been associated with clinically overt cardiovascular diseases including HF [17].

The TCR signaling pathway plays a key role in functioning of $\mathrm{T}$ cells and $\mathrm{T}$ cell recruitment into the $\mathrm{LV}$ appears to contribute negatively to pathological cardiac remodeling in HF. In end-stage HF patients, T cells activated and infiltrated the failing heart, playing a part in the development of LV remodeling and negatively affected cardiac function through mechanisms involving cytokine release and induction of cardiac fibrosis and hypertrophy [18]

The CXCR4 receptor and its ligand CXCL12 play a crucial role in the homing of stem and progenitor cells in the bone marrow and control their mobilization into peripheral blood and tissues after tissue injury or stress. The CXCR4 system is expressed in cardiac myocytes and the CXCL12/CXCR4 axis is involved in chemotaxis, cell arrest, angiogenesis and cell survival [19].

Our NGS analysis demonstrated that such pathophysiological signaling pathways were activated; therefore, it is very important to examine the extent to which statin explained the significance of the clues afforded by altered gene expression across the three transcriptome pathways uncovered by NGS analysis. Of these, SHC1 which was the most highly expressed overlapping gene identified in pathway analysis, encodes a molecular adapter protein which exists in three isoforms $\mathrm{p} 46^{\text {Shc }}, \mathrm{p} 52^{\text {Shc }}$ and $\mathrm{p} 66^{\text {Shc }}$. Both $\mathrm{p} 46^{\text {Shc }}$ and $\mathrm{p} 52^{\text {Shc }}$ promote cell proliferation and differentiation via Ras and MAP kinases and $\mathrm{p} 66^{\text {Shc }}$ has been proposed to be a key factor functioning as a redox enzyme 
Citation: Wada A, Matsumoto T, Takayama T, Suzaki M, Taniguchi A, et al. (2018) New Insights in to Molecular Therapeutic Mechanism of Statin Action in Heart Failure: Use of High-Throughput Transcriptome Analysis. Cardiovasc Pharm Open Access 6: 232. doi: 10.4172/23296607.1000232

Page 5 of 6

implicated in mitochondrial reactive oxygen species (ROS) generation and translation of oxidative signals into apoptosis [20]. In the present study, reduced $\mathrm{SHC1}$ expression in the presence of statin indicated that the latter may protect against myocardial hypertrophy and apoptosis mediated by ROS-induced injury in HF.

Cardiac hypertrophy is an adaptive response of the heart to several cardiac forms of overload. In the myocardium, the small GTP-binding proteins Ras, Rho and Rac and oxidative stress are involved in the hypertrophic response. VAVl activates RhoGTPases by facilitating the exchange of GDP for GTP. VAVl is expressed in hematopoietic cells, controlling functions such as the antigen response of $\mathrm{T}$ cells, phagocytosis by macrophages and superoxide production and is a critical component of the TCR signaling cascade [21]. VAV1 deficiency reduces TCR-induced proliferation, intracellular $\mathrm{Ca}^{2+}$ flux and cytokine secretion.

ELK1 is a member of the ternary complex factor subfamily and functions as a nuclear transcriptional activator via its association with serum response factor (SRF). The majority of SRF target genes are involved in cell growth, migration, cytoskeletal organization and myogenesis and the primary action is thought to involve activation of immediate-early genes in response to pressure-overloaded, angiotensin II-induced myocyte hypertrophy [22]. Taken together, our study indicated that pitavastatin may maintain adequate cardiac levels of VAV1 and ELK1 and preserve cardiac homeostasis against the hypertrophic response and ROS and, in part, result in amelioration of LV diastolic dysfunction.

SRC (including isoforms SRC-1 3) has evolved as a pleiotropic coordinator of diverse physiological systems ranging from immune protection to reproduction [23]. The SRC family also plays important global roles in control of metabolic homeostasis and energy balance. The heart, which requires a constant supply of fuel, relies mainly on the use of fatty acids for ATP production, but can supplement this with glucose utilization during periods of increased workload such as HF. Ablation of SRC-2 results in a clear drop in the molar sum of all cardiac amino acid levels in the fasted state, suggesting that SRC-2 maintains amino acid metabolism in the heart [24]. In the present study, pitavastatin suppressed SRC gene up-regulation in HF, indicating that it potentially stabilized cardiac energy metabolism balance.

FLNA anchors the actin cytoskeleton to adhesion receptors and null mutations in FLNA cause structural defects in heart [25]. Loss of FLNA leads to a decrease of tissue inhibitor of metalloproteinase 2 (TIMP-2) secretions, an increase of matrix metalloproteinase2 (MMP2) activities and increased peri-cellular matrix degradation. Activated FLNA appears to act to suppress ECM degradation to compensate for LV ECM remodeling in HF [26]. In the present study, we did not investigate changes in MMP or TIMP expression in failing heart. However, pitavastatin decreased collagen deposition significantly; therefore, it improved the ECM balance in HF, partly through reduction of FLNA expression. Because our NGS analysis showed that the ECM pathway was significantly up-regulated in HF, we further examined changes in fibrotic molecular markers to provide additional information on regulation of cardiac stiffness in failing myocardium. In the present study, we confirmed that pitavastatin not only reduced gene expression of COL1A1 and COL3A1, but also COL6A1 and decreased the deposition of collagen. Type I and type III collagen are major constituents of the cardiac ECM that provides structural and mechanical support to the heart and acts as signaling conduit between myocardial cells. In contrast, collagen VI forms a microfilament network that organizes the fibrilin collagens and anchors these to the basement membrane [27]. A change in collagen architecture, with abnormal alignment and/or endo myocardial fibrosis, would be responsible for an increase in myocardial stiffness; thus beneficial effects of statin on diastolic properties could partially result from altered collagen turnover and deposition in the failing heart.

There are some limitations in relation to the present study. Since we attempted to clarify which types of gene pathways mainly contribute to the development of HF, we did not directly compare the changes in gene expression among our three experimental groups. We need to undertake a comparative RNA-seq study of a station in HF in the future. Second, we only evaluated up-regulated pathway genes; however, 25 genes were down-regulated in HF. Down-regulated pathway analysis was previously reported to be involved in cardiac muscle contraction and metabolism in cardiac hypertrophy [28]. We also need to identify the nature of the effects exerted by down-regulated genes on cardiac function in statin treatment.

\section{Conclusion}

In summary, our transcriptome analysis carried out through RNA-seq and pathway analysis has provided important insights into comprehensive gene expression changes in failing hearts. The clinical significance of the pleiotropic effects of statins in the cardiovascular system remains controversial. However, our identified genes play pivotal roles in cell proliferation, inflammation, ROS production, energy metabolism and EMC balance and pitavastatin ameliorated diastolic properties, accompanied by changes in expression of our identified genes in HF. High-throughput transcriptome analysis can provide new insights into cellular mechanisms underlying HF and into potential targets for therapeutic intervention.

\section{Acknowledgment}

We thank Ms. Fumiko Kimura and Mr. Takefumi Yamamoto (Central Research Laboratory, Shiga University of medical Science) for assistance with qRT-PCR and are also grateful to Kowa Pharmaceutical Co., Ltd., Japan for supplying pitavastatin.

\section{Sources of Funding}

This study received grants from MSD Life Science Foundation, the Mochida Memorial Foundation for Medical and Pharmaceutical Research and the Takeda Medical Research Foundation. The funding sources had no influence on the design of the study or in the collection, analysis, or interpretation of the data.

\section{References}

1. Dohke T, Wada A, Isono T, Fujii M, Yamamoto T, et al. (2006) Proteomic analysis reveals significant alternations of cardiac small heat shock protein expression in congestive heart failure. J Card Fail 12: 77-84.

2. Churko JM, Mantalas GL, Snyder MP, Wu JC (2013) Overview of high throughput sequencing technologies to elucidate molecular pathways in cardiovascular diseases. Circ Res 112: 1613-23.

3. Matkovich SJ, Zhang Y, Van Booven DJ, Dorn GW (2010) Deep mRNA sequencing for in vivo functional analysis of cardiac transcriptional regulators: Application to Galphaq. Circ Res 106: 1459-67.

4. Isono T, Matsumoto T, Wada A, Suzaki M, Chano T (2012) A global transcriptome analysis of a dog model of congestive heart failure with the human genome as a reference. J Card Fail 18: 872-8.

5. Hartupee J, Mann DL (2013) Positioning of inflammatory biomarkers in the heart failure landscape. J Cardiovasc Transl Res 6: 485-92

6. Go AS, Lee WY, Yang J, Lo JC, Gurwitz JH (2006) Statin therapy and risks for death and hospitalization in chronic heart failure. JAMA 296: 2105-11.

7. Kjekshus J, Apetrei E, Barrios V, Bohm M, Cleland JG, et al. (2007) Rosuvastatin in older patients with systolic heart failure. N Engl J Med 357: 2248-61.

8. Tavazzi L, Maggioni AP, Marchioli R, Barlera S, Franzosi MG, et al. (2008) Effect of rosuvastatin in patients with chronic heart failure (the GISSI-HF trial): a randomized, double-blind, placebo-controlled trial. Lancet 372: 1231-9. 
Citation: Wada A, Matsumoto T, Takayama T, Suzaki M, Taniguchi A, et al. (2018) New Insights in to Molecular Therapeutic Mechanism of Statin Action in Heart Failure: Use of High-Throughput Transcriptome Analysis. Cardiovasc Pharm Open Access 6: 232. doi: 10.4172/23296607.1000232

Page 6 of 6

9. Preiss D, Campbell RT, Murray HM, Ford I, Packard CJ, et al. (2015) The effect of statin therapy on HF events: a collaborative meta-analysis of unpublished data from major randomized trials. Eur Heart J 36: 1536-46.

10. Wada A, Tsutamoto T, Matsuda Y, Kinoshita M (1994) Cardiorenal and neurohumoral effects of endogenous atrial natriuretic peptide in dogs with severe congestive heart failure using a specific antagonist for guanylate cyclase-coupled receptors. Circulation 89: 2232-40.

11. Wada A, Tsutamoto T, Ohnishi M, Sawaki M, Fukai D, et al. (1999) Effects of a specific endothelin-converting enzyme inhibitor on cardiac, renal, and neurohumoral functions in congestive heart failure: Comparison of effects with those of endothelin a receptor antagonism. Circulation 99: 570-577.

12. Takayama T, Wada A, Tsutamoto T, Ohnishi M, Fujii M, et al. (2004) Contribution of vascular $\mathrm{NAD}(\mathrm{P}) \mathrm{H}$ oxidase to endothelial dysfunction in heart failure and the therapeutic effects of HMG-CoA reductase inhibitor. Circ J 68: 1067-75.

13. Fujii M, Wada A, Tsutamoto T, Ohnishi M, Isono T, et al. (2002) Bradykinin improves left ventricular diastolic function under long-term angiotensinconverting enzyme inhibition in heart failure. Hypertension 39: 952-7.

14. Matsumoto T, Wada A, Tsutamoto T, Ohnishi M, Isono T, et al. (2003) Chymase inhibition prevents cardiac fibrosis and improves diastolic dysfunction in the progression of heart failure. Circulation. 107: 2555-2558.

15. Alehagen U, Benson L, Edner M, Dahlstrom U, Lund LH (2015) Association between use of statins and mortality in patients with heart failure and ejection fraction of $\geq 50$. Circ Heart Fail 8: 862-70.

16. Fiedler U, Augustin HG (2006) Angiopoietins: A link between angiogenesis and inflammation. Trends Immunol 27: 552-8.

17. Chong AY, Caine GJ, Freestone B, Blann AD, Lip GY (2004) Plasma angiopoietin-1, angiopoietin-2, and angiopoietin receptor tie-2 levels in congestive heart failure. J Am Coll Cardiol 43: 423-8.

18. Nevers T, Salvador AM, Grodecki-Pena A, Knapp A, Velázquez F, et al. (2015) Left ventricular T-Cell recruitment contributes to the pathogenesis of heart failure. Circ Heart Fail 8: 776-787.
19. Saxena A1, Fish JE, White MD, Yu S, Smyth JW, et al. (2008) Stromal cellderived factor-1alpha is cardioprotective after myocardial infarction. Circulation 117: 2224-31.

20. Obreztchikova M, Elouardighi H, Ho M, Wilson BA, Gertsberg Z, et al. (2006) Distinct signaling functions for Shc isoforms in the heart. J Biol Chem 281: 20197-204.

21. Chrencik JE, Brooun A, Zhang H, Mathews II, Hura GL, et al. (2008) Structura basis of guanine nucleotide exchange mediated by the T-cell essential Vav1. J Mol Biol 380: 828-843.

22. Vickers ER, Kasza A, Kurnaz IA, Seifert A, Zeef LA, et al. (2004) Ternary complex factor-serum response factor complex-regulated gene activity is required for cellular proliferation and Inhibition of apoptotic cell death. Mol Cell Biol 24: 10340-51.

23. York B, Bert W O'Malley (2010) Steroid receptor coactivator (SRC) family: Masters of systems biology. J Biol Chem 285: 38743-38750.

24. York B, Sagen JV, Tsimelzon A, Louet JF, Chopra AR, et al. (2013) Research resource: Tissue- and pathway-specific metabolomic profiles of the steroid receptor coactivator (SRC) family. Mol Endocrinol 27: 366-80.

25. Bandaru S, Gronros J, Redfors B, Cil C, Pazooki D, et al. (2015) Deficiency of filamin $A$ in endothelial cells impairs left ventricular remodelling after myocardial infarction. Cardiovasc Res 105: 151-9.

26. Baldassarre M, Razinia Z, Brahme NN, Buccione R, Calderwood DA (2012) Filamin A controls matrix metalloproteinase activity and regulates cell invasion in human fibrosarcoma cells. J Cell Sci 125: 3858-69.

27. Keene DR, Engvall E, Glanville RW (1988) Ultrastructure of type VI collagen in human skin and cartilage suggests an anchoring function for this filamentous network. J Cell Biol 107: 1995-2006.

28. Yang KC, Yamada KA, Patel AY, Topkara VK, George I, et al. (2014) Deep RNA sequencing reveals dynamic regulation of myocardial noncoding RNAs in failing human heart and remodeling with mechanical circulatory support. Circulation 129: 1009-21. 